Electronic Supporting Information for:

\title{
Understanding the Formation of Salt-Inclusion Phases: An Enhanced Flux Growth Method for the Targeted Synthesis of Salt-Inclusion Cesium Halide Uranyl Silicates
}

\author{
Gregory Morrison, Mark D. Smith, Hans-Conrad zur Loye*
}


Table S1 Select interatomic distances $(\AA)$ and bond valence sums for $\left[\mathrm{Cs}_{3} \mathrm{~F}\right]\left[\left(\mathrm{UO}_{2}\right)\left(\mathrm{Si}_{4} \mathrm{O}_{10}\right)\right](\mathbf{1})$.

\begin{tabular}{|c|c|c|c|c|c|}
\hline Interaction & Distance & Interaction & Distance & Interaction & Distance \\
\hline $\mathrm{U}(1)-\mathrm{O}(2) \mathrm{x} 2$ & $1.815(4)$ & $\mathrm{Si}(1)-\mathrm{O}(3)$ & $1.584(3)$ & $\mathrm{F}(1)-\mathrm{Cs}(1) \times 2$ & $2.8516(4)$ \\
\hline $\mathrm{U}(1)-\mathrm{O}(3) \times 4$ & $2.242(3)$ & $\mathrm{Si}(1)-\mathrm{O}(5)$ & $1.6109(13)$ & $F(1)-C s(2) \times 4$ & $3.4345(6)$ \\
\hline \multirow[t]{9}{*}{ BVS U(1) } & 5.92 & $\mathrm{Si}(1)-\mathrm{O}(4)$ & $1.6149(16)$ & BVS F(1) & 0.79 \\
\hline & & $\mathrm{Si}(1)-\mathrm{O}(1)$ & $1.6175(16)$ & $\mathrm{F}(2)-\mathrm{Cs}(1)$ & $2.87(2)$ \\
\hline & & BVS Si(1) & 4.19 & $\mathrm{~F}(2)-\mathrm{Cs}(1)$ & $2.93(2)$ \\
\hline & & & & $\mathrm{F}(2)-\mathrm{Cs}(2) \times 2$ & $3.081(13)$ \\
\hline & & & & BVS F(2) & 0.79 \\
\hline & & & & $\mathrm{F}(3)-\mathrm{Cs}(2) \times 2$ & $2.79(2)$ \\
\hline & & & & $\mathrm{F}(3)-\mathrm{Cs}(1)$ & $3.01(4)$ \\
\hline & & & & $\mathrm{F}(3)-\mathrm{Cs}(1)$ & $3.09(4)$ \\
\hline & & & & BVS F(3) & 0.99 \\
\hline
\end{tabular}


Table S2 Select interatomic distances $(\AA)$ and bond valence sums for $\left[\mathrm{Cs}_{2} \mathrm{Cs}_{5} \mathrm{~F}\right]\left[\left(\mathrm{UO}_{2}\right)_{3}\left(\mathrm{Si}_{2} \mathrm{O}_{7}\right)_{2}\right]$ (2).

\begin{tabular}{llllll} 
Interaction & Distance & Interaction & Distance & Interaction & Distance \\
\hline $\mathrm{U}(1)-\mathrm{O}(3) \times 2$ & $1.838(6)$ & $\mathrm{Si}(1)-\mathrm{O}(8)$ & $1.615(6)$ & $\mathrm{F}(1)-\mathrm{Cs}(2)$ & $2.908(14)$ \\
$\mathrm{U}(1)-\mathrm{O}(9) \times 2$ & $2.210(5)$ & $\mathrm{Si}(1)-\mathrm{O}(10)$ & $1.619(5)$ & $\mathrm{F}(1)-\mathrm{Cs}(2)$ & $2.911(14)$ \\
$\mathrm{U}(1)-\mathrm{O}(8) \times 2$ & $2.218(5)$ & $\mathrm{Si}(1)-\mathrm{O}(5)$ & $1.623(6)$ & $\mathrm{F}(1)-\mathrm{Cs}(4)$ & $2.930(13)$ \\
$\mathbf{B V S ~ U}(\mathbf{1})$ & $\mathbf{5 . 9 4}$ & $\mathrm{Si}(1)-\mathrm{O}(2)$ & $1.647(5)$ & $\mathrm{F}(1)-\mathrm{Cs}(3)$ & $3.105(17)$ \\
$\mathrm{U}(2)-\mathrm{O}(1)$ & $1.839(6)$ & $\mathbf{B V S ~ S i}(\mathbf{1})$ & $\mathbf{3 . 9 8}$ & $\mathrm{F}(1)-\mathrm{Cs}(3)$ & $3.143(16)$ \\
$\mathrm{U}(2)-\mathrm{O}(4)$ & $1.844(6)$ & $\mathrm{Si}(2)-\mathrm{O}(6)$ & $1.602(6)$ & $\mathbf{B V S} \mathbf{F}(\mathbf{1})$ & $\mathbf{0 . 9 7}$ \\
$\mathrm{U}(2)-\mathrm{O}(6)$ & $2.183(6)$ & $\mathrm{Si}(2)-\mathrm{O}(7)$ & $1.610(6)$ & & \\
$\mathrm{U}(2)-\mathrm{O}(10)$ & $2.206(5)$ & $\mathrm{Si}(2)-\mathrm{O}(9)$ & $1.611(6)$ & & \\
$\mathrm{U}(2)-\mathrm{O}(7)$ & $2.229(6)$ & $\mathrm{Si}(2)-\mathrm{O}(2)$ & $1.642(5)$ & & \\
$\mathrm{U}(2)-\mathrm{O}(5)$ & $2.245(5)$ & $\mathbf{B V S ~ S i}(2)$ & $\mathbf{4 . 0 9}$ & & \\
$\mathbf{B V S ~ U}(2)$ & $\mathbf{5 . 9 1}$ & & &
\end{tabular}


Table S3 Select interatomic distances $(\AA)$ and bond valence sums for $\left[\mathrm{Cs}_{2} \mathrm{Cs}_{5} \mathrm{~F}\right]\left[\left(\mathrm{UO}_{2}\right)_{2}\left(\mathrm{Si}_{6} \mathrm{O}_{17}\right)\right]$ (3).

\begin{tabular}{|c|c|c|c|c|c|}
\hline Interaction & Distance & Interaction & Distance & Interaction & Distance \\
\hline $\mathrm{U}(1)-\mathrm{O}(1)$ & $1.822(11)$ & $\mathrm{Si}(1)-\mathrm{O}(6)$ & $1.572(15)$ & $\mathrm{F}(1)-\operatorname{Cs}(2) \times 2$ & $3.1165(17)$ \\
\hline $\mathrm{U}(1)-\mathrm{O} 10)$ & $1.833(15)$ & $\mathrm{Si}(1)-\mathrm{O}(8)$ & $1.587(6)$ & $\mathrm{F}(1)-\mathrm{Cs}(4 \mathrm{a}) \times 2$ & $3.166(4)$ \\
\hline $\mathrm{U}(1)-\mathrm{O}(9)$ & $2.211(13)$ & $\mathrm{Si}(1)-\mathrm{O}(4)$ & $1.632(12)$ & $\mathrm{F}(1)-\mathrm{Cs}(3)$ & $3.31(3)$ \\
\hline $\mathrm{U}(1)-\mathrm{O}(11)$ & $2.232(12)$ & $\mathrm{Si}(1)-\mathrm{O}(5)$ & $1.645(13)$ & $\mathrm{F}(1)-\mathrm{Cs}(3)$ & $3.87(3)$ \\
\hline $\mathrm{U}(1)-\mathrm{O}(7)$ & $2.245(13)$ & BVS Si(1) & 4.18 & BVS F(1) & 0.61 \\
\hline $\mathrm{U}(1)-\mathrm{O}(3)$ & $2.268(15)$ & $\mathrm{Si}(2)-\mathrm{O}(7)$ & $1.601(14)$ & & \\
\hline \multirow[t]{9}{*}{ BVS U(1) } & 5.86 & $\mathrm{Si}(2)-\mathrm{O}(2)$ & $1.616(12)$ & & \\
\hline & & $\mathrm{Si}(2)-\mathrm{O}(9)$ & $1.622(14)$ & & \\
\hline & & $\mathrm{Si}(2)-\mathrm{O}(5)$ & $1.624(13)$ & & \\
\hline & & BVS Si(2) & 4.09 & & \\
\hline & & $\mathrm{Si}(3)-\mathrm{O}(11)$ & $1.578(13)$ & & \\
\hline & & $\mathrm{Si}(3)-\mathrm{O}(3)$ & $1.609(16)$ & & \\
\hline & & $\mathrm{Si}(3)-\mathrm{O}(4)$ & $1.610(13)$ & & \\
\hline & & $\mathrm{Si}(3)-\mathrm{O}(2)$ & $1.657(12)$ & & \\
\hline & & BVS Si(3) & 4.13 & & \\
\hline
\end{tabular}


Table S4 Select interatomic distances $(\AA)$ and bond valence sums for $\left[\mathrm{Cs}_{9} \mathrm{Cs}_{6} \mathrm{Cl}\right]\left[\left(\mathrm{UO}_{2}\right)_{7}\left(\mathrm{Si}_{6} \mathrm{O}_{17}\right)_{2}\left(\mathrm{Si}_{4} \mathrm{O}_{12}\right)\right](4)$.

\begin{tabular}{|c|c|c|c|c|c|}
\hline Interaction & Distance & Interaction & Distance & Interaction & Distance \\
\hline $\mathrm{U}(1)-\mathrm{O}(10)$ & $1.806(12)$ & $\mathrm{Si}(1)-\mathrm{O}(21)$ & $1.581(14)$ & $\mathrm{Si}(5)-\mathrm{O}(27)$ & $1.570(15)$ \\
\hline $\mathrm{U}(1)-\mathrm{O}(16)$ & $1.809(11)$ & $\mathrm{Si}(1)-\mathrm{O}(1)$ & $1.614(13)$ & $\mathrm{Si}(5)-\mathrm{O}(19)$ & $1.576(15)$ \\
\hline $\mathrm{U}(1)-\mathrm{O}(27)$ & $2.207(14)$ & $\mathrm{Si}(1)-\mathrm{O}(7)$ & $1.614(14)$ & $\mathrm{Si}(5)-\mathrm{O}(11)$ & $1.618(15)$ \\
\hline $\mathrm{U}(1)-\mathrm{O}(12)$ & $2.215(14)$ & $\mathrm{Si}(1)-\mathrm{O}(5)$ & $1.636(13)$ & $\mathrm{Si}(5)-\mathrm{O}(13)$ & $1.622(14)$ \\
\hline $\mathrm{U}(1)-\mathrm{O}(26)$ & $2.238(13)$ & BVS Si(1) & 4.15 & BVS Si(5) & 4.51 \\
\hline $\mathrm{U}(1)-\mathrm{O}(18)$ & $2.241(13)$ & $\mathrm{Si}(2)-\mathrm{O}(6)$ & $1.577(13)$ & $\mathrm{Si}(6)-\mathrm{O}(12)$ & $1.578(16)$ \\
\hline BVS U(1) & 6.06 & $\mathrm{Si}(2)-\mathrm{O}(9)$ & $1.618(13)$ & $\mathrm{Si}(6)-\mathrm{O}(22)$ & $1.584(15)$ \\
\hline $\mathrm{U}(2)-\mathrm{O}(25)$ & $1.777(13)$ & $\mathrm{Si}(2)-\mathrm{O}(1)$ & $1.627(14)$ & $\mathrm{Si}(6)-\mathrm{O}(15)$ & $1.635(14)$ \\
\hline $\mathrm{U}(2)-\mathrm{O}(23)$ & $1.827(9)$ & $\mathrm{Si}(2)-\mathrm{O}(5)$ & $1.628(14)$ & $\mathrm{Si}(6)-\mathrm{O}(13)$ & $1.640(14)$ \\
\hline $\mathrm{U}(2)-\mathrm{O}(3)$ & $2.229(14)$ & BVS Si(2) & 4.13 & BVS Si(6) & 4.36 \\
\hline $\mathrm{U}(2)-\mathrm{O}(4)$ & $2.232(13)$ & $\mathrm{Si}(3)-\mathrm{O}(3)$ & $1.571(14)$ & $\mathrm{Si}(7)-\mathrm{O}(15)$ & $1.581(15)$ \\
\hline $\mathrm{U}(2)-\mathrm{O}(21)$ & $2.254(13)$ & $\mathrm{Si}(3)-\mathrm{O}(18)$ & $1.603(13)$ & $\mathrm{Si}(7)-\mathrm{O}(11)$ & $1.588(15)$ \\
\hline $\mathrm{U}(2)-\mathrm{O}(6)$ & $2.281(12)$ & $\mathrm{Si}(3)-\mathrm{O}(24)$ & $1.613(15)$ & $\mathrm{Si}(7)-\mathrm{O}(31 \mathrm{~A})$ & $1.61(2)$ \\
\hline BVS U(2) & 5.97 & $\mathrm{Si}(3)-\mathrm{O}(8)$ & $1.634(12)$ & $\mathrm{Si}(7)-\mathrm{O}(2)$ & $1.632(10)$ \\
\hline $\mathrm{U}(3)-\mathrm{O}(28)$ & $1.804(12)$ & BVS Si(3) & 4.22 & $\mathrm{Si}(7)-\mathrm{O}(31 \mathrm{~B})$ & $1.64(2)$ \\
\hline $\mathrm{U}(3)-\mathrm{O}(17)$ & $1.891(12)$ & $\mathrm{Si}(4)-\mathrm{O}(4)$ & $1.590(13)$ & BVS Si(7) & 4.39 \\
\hline $\mathrm{U}(3)-\mathrm{O}(7)$ & $2.175(14)$ & $\mathrm{Si}(4)-\mathrm{O}(26)$ & $1.594(15)$ & $\mathrm{Si}(8)-\mathrm{O}(14)$ & $1.601(13)$ \\
\hline $\mathrm{U}(3)-\mathrm{O}(9)$ & $2.177(13)$ & $\mathrm{Si}(4)-\mathrm{O}(14)$ & $1.621(13)$ & $\mathrm{Si}(8)-\mathrm{O}(24)$ & $1.601(14)$ \\
\hline $\mathrm{U}(3)-\mathrm{O}(22)$ & $2.241(13)$ & $\mathrm{Si}(4)-\mathrm{O}(8)$ & $1.644(12)$ & $\mathrm{Si}(8)-\mathrm{O}(30 \mathrm{~A})$ & $1.62(4)$ \\
\hline $\mathrm{U}(3)-\mathrm{O}(19)$ & $2.243(15)$ & BVS Si(4) & 4.14 & $\mathrm{Si}(8)-\mathrm{O}(2)$ & $1.627(11)$ \\
\hline BVS U(3) & 5.93 & & & $\mathrm{Si}(8)-\mathrm{O}(30 \mathrm{~B})$ & $1.65(4)$ \\
\hline $\mathrm{U}(4)-\mathrm{O}(29 \mathrm{~A}) \mathrm{x} 2$ & $1.85(2)$ & & & BVS Si(8) & 4.27 \\
\hline $\mathrm{U}(4)-\mathrm{O}(31 \mathrm{~A}) \mathrm{x} 2$ & $2.25(4)$ & & & $\mathrm{Cl}(1)-\mathrm{Cs}(5) \times 2$ & $3.3285(11)$ \\
\hline $\mathrm{U}(4)-\mathrm{O}(30 \mathrm{~A}) \times 2$ & $2.26(2)$ & & & $\mathrm{Cl}(1)-\mathrm{Cs}(6) \times 2$ & $3.5433(12)$ \\
\hline BVS U(4) & 5.65 & & & $\mathrm{Cl}(1)-\mathrm{Cs}(1) \times 2$ & $3.7019(15)$ \\
\hline $\mathrm{U}(4)-\mathrm{O}(29 \mathrm{~B}) \mathrm{x} 2$ & $1.80(3)$ & & & BVS Cl(1) & 0.90 \\
\hline $\mathrm{U}(4)-\mathrm{O}(30 \mathrm{~B}) \mathrm{x} 2$ & $2.12(4)$ & & & & \\
\hline $\mathrm{U}(4)-\mathrm{O}(31 \mathrm{~B}) \mathrm{x} 2$ & $2.22(2)$ & & & & \\
\hline BVS U(4) & 6.44 & & & & \\
\hline
\end{tabular}


Table S5: Classification of all reported uranyl silicates based on their silicate, uranium, and overall dimensionality. (Note:

Reference numbers do not correspond to those in the manuscript.)

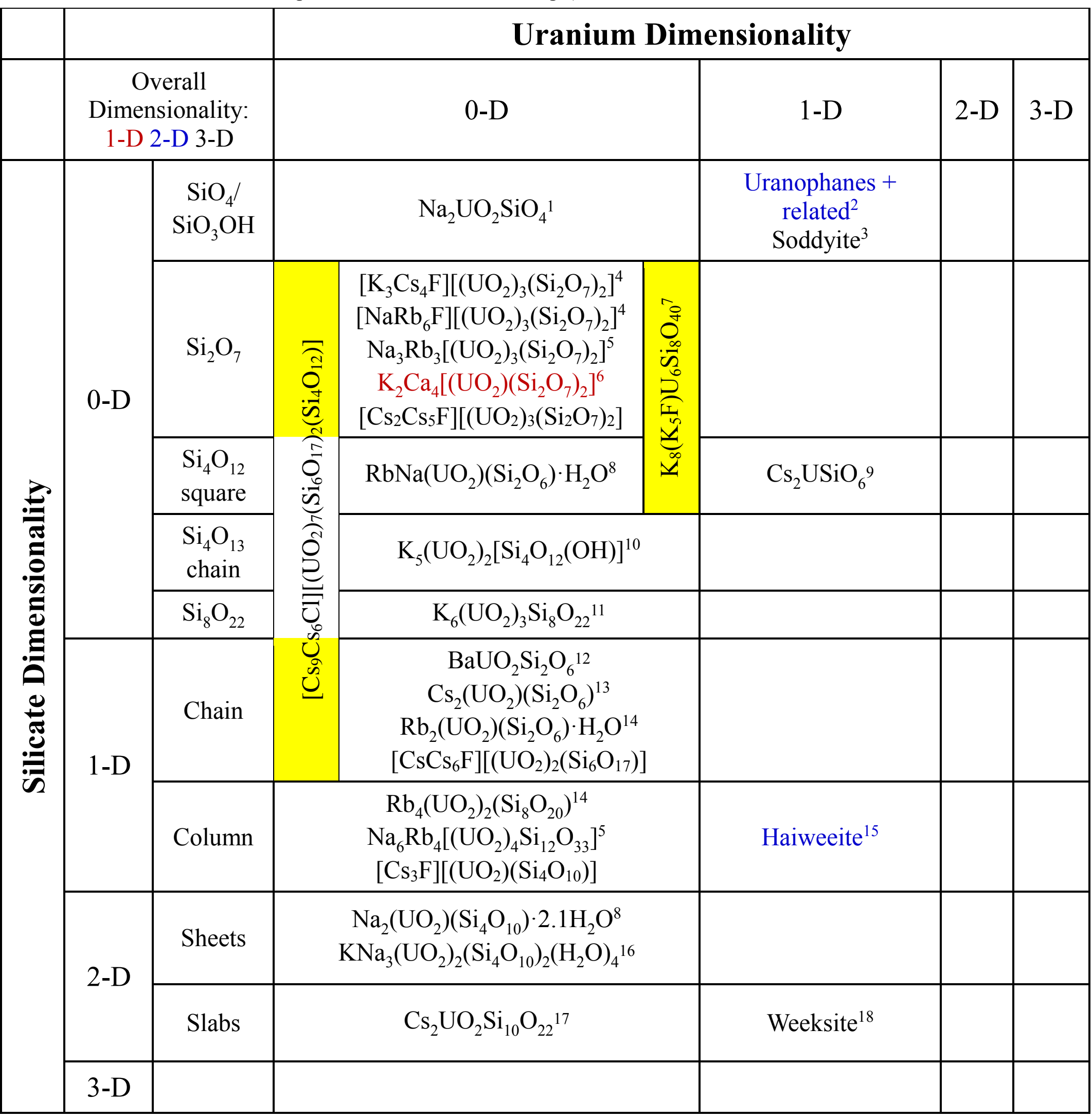


Figure S1 Powder X-ray diffraction patterns for each reaction listed in Figure 1. Each powder diffraction pattern was analyzed for the presence of each salt-inclusion and non-salt-inclusion cesium uranyl silicate along with for the presence of $\mathrm{AgCl}$.

a) $\mathrm{UO}_{2}$ 1:1

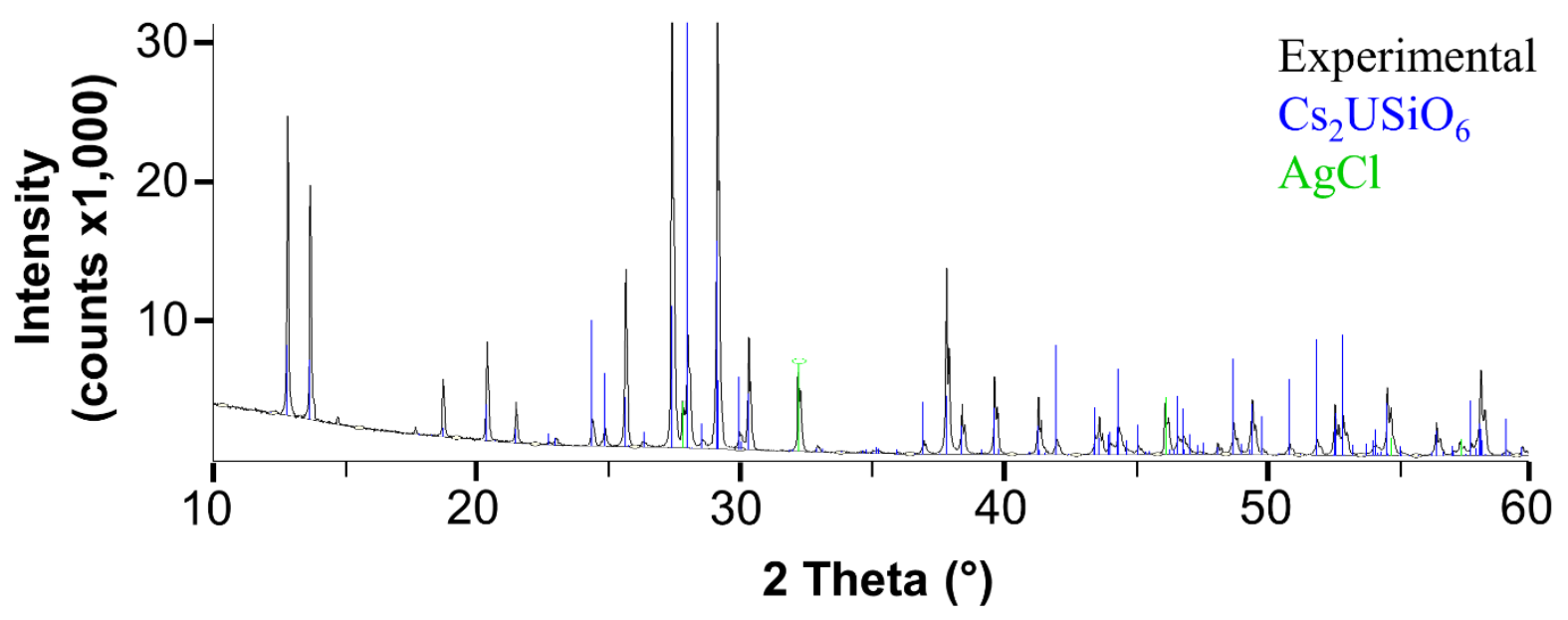

b) $\mathbf{U O}_{2} 1: 2$

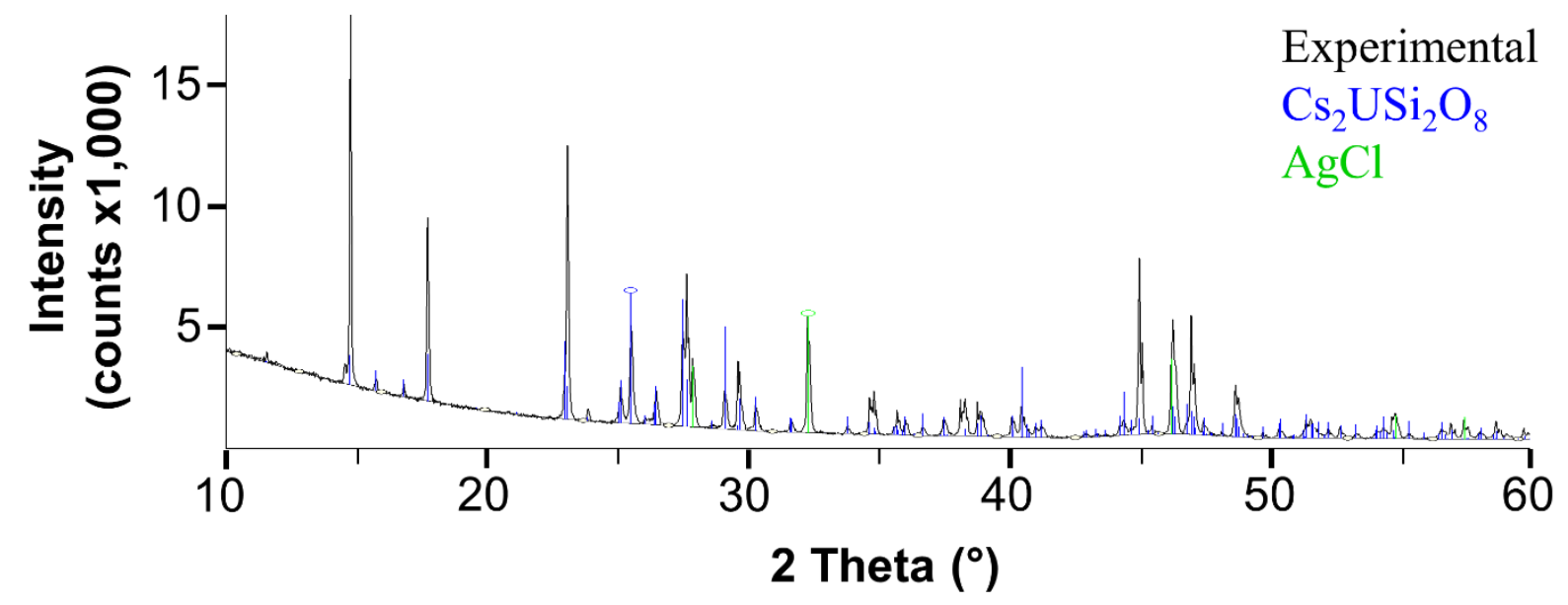

c) $\mathrm{UO}_{2} 1: 3$

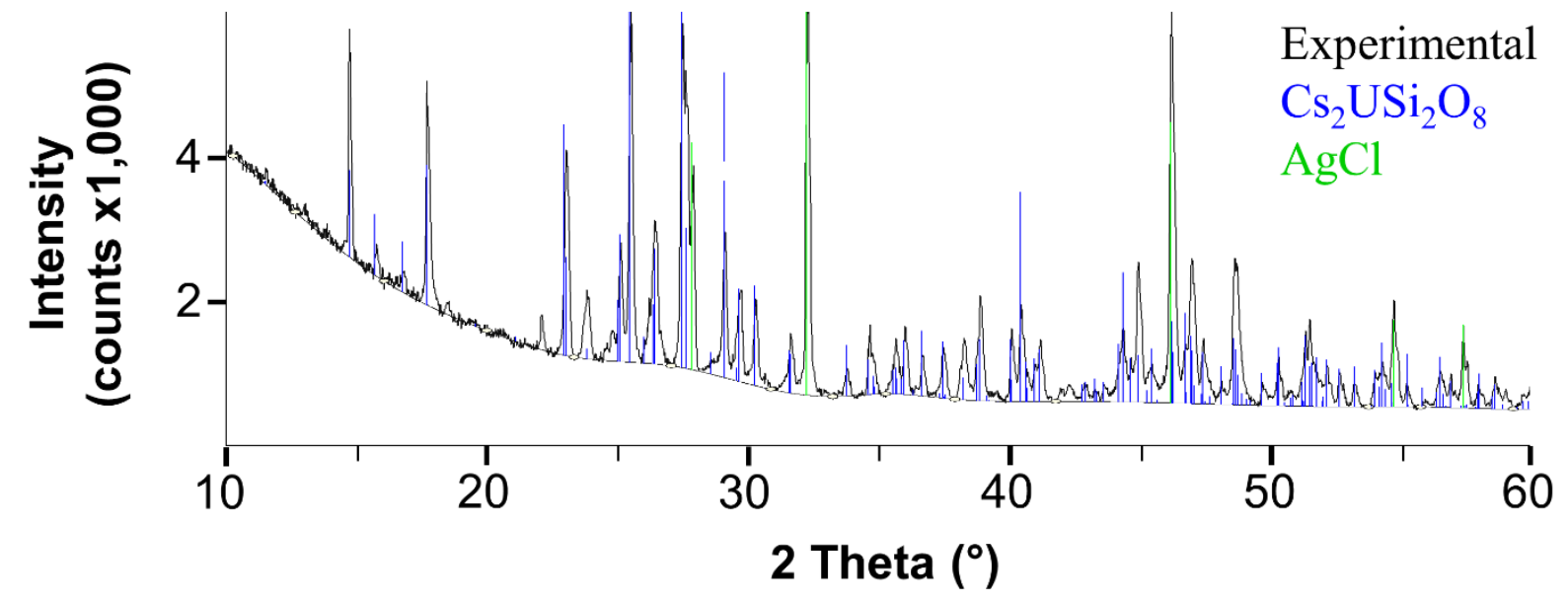


d) $\mathrm{UO}_{2}$ 1:4

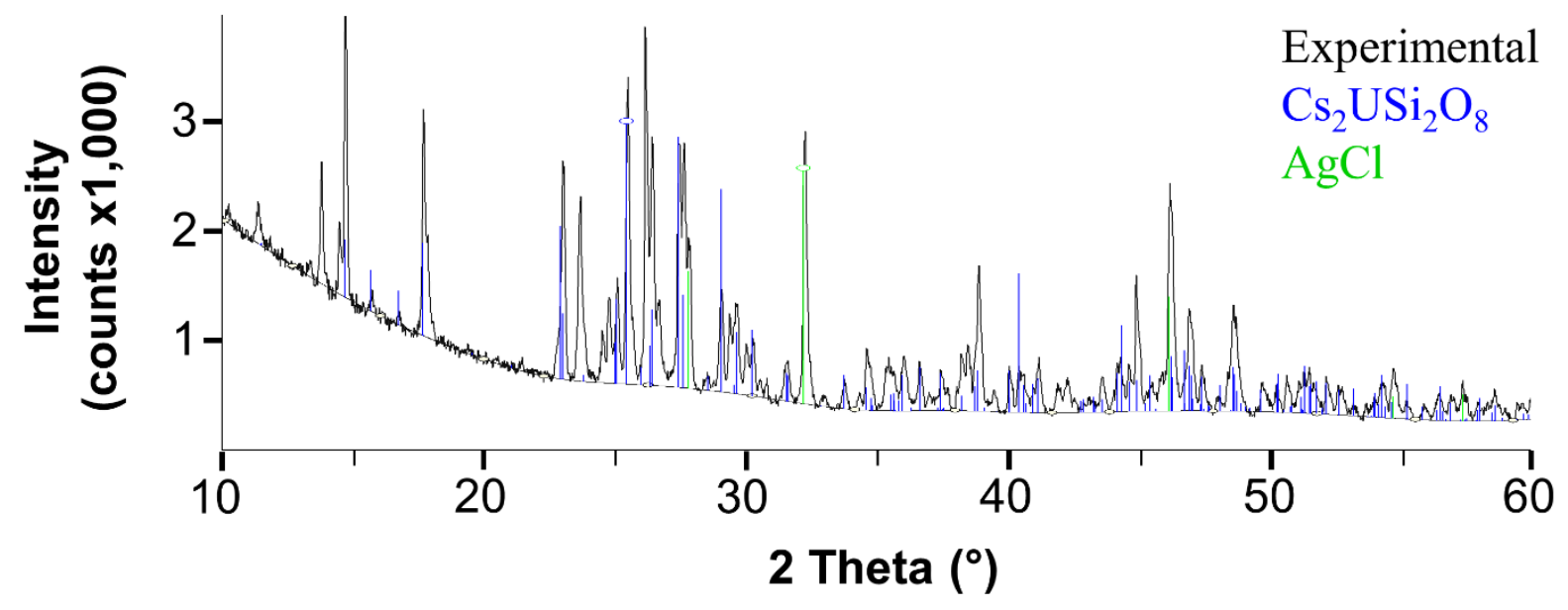

e) $\mathbf{U O}_{2} \mathbf{1 : 8}$

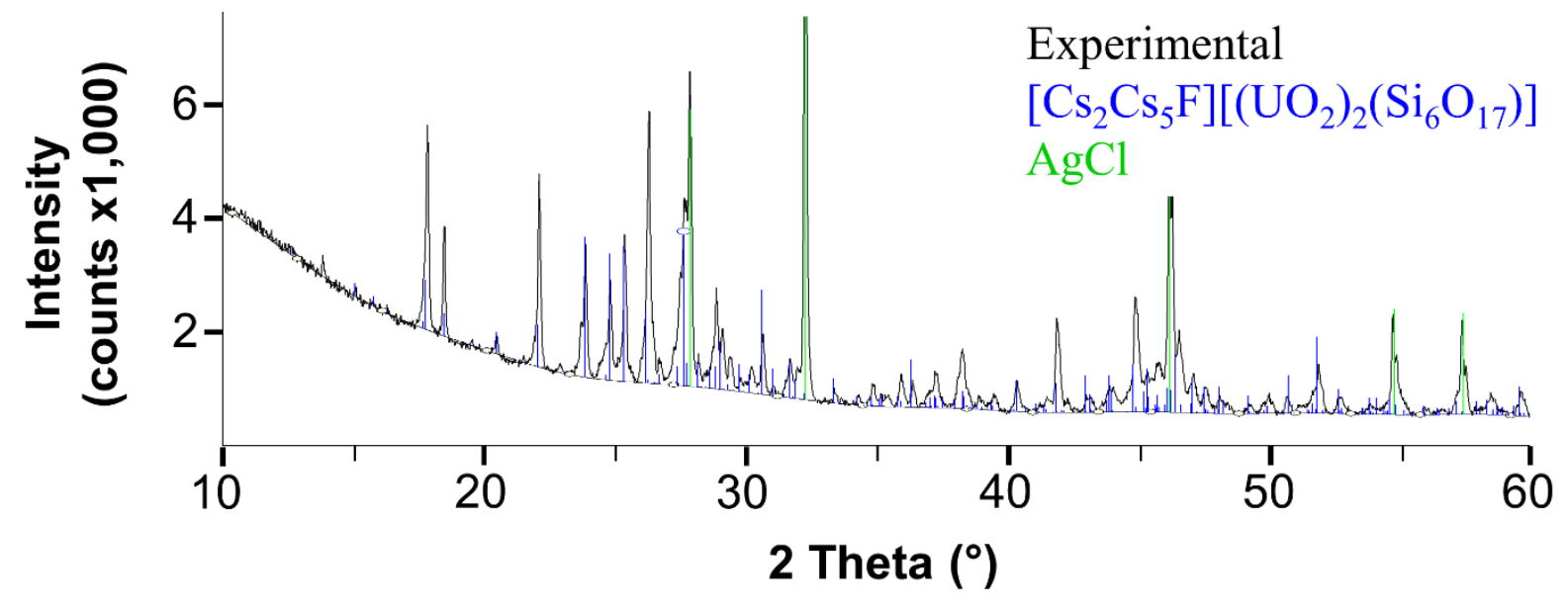

f) $\mathrm{UO}_{2} 1: 12$

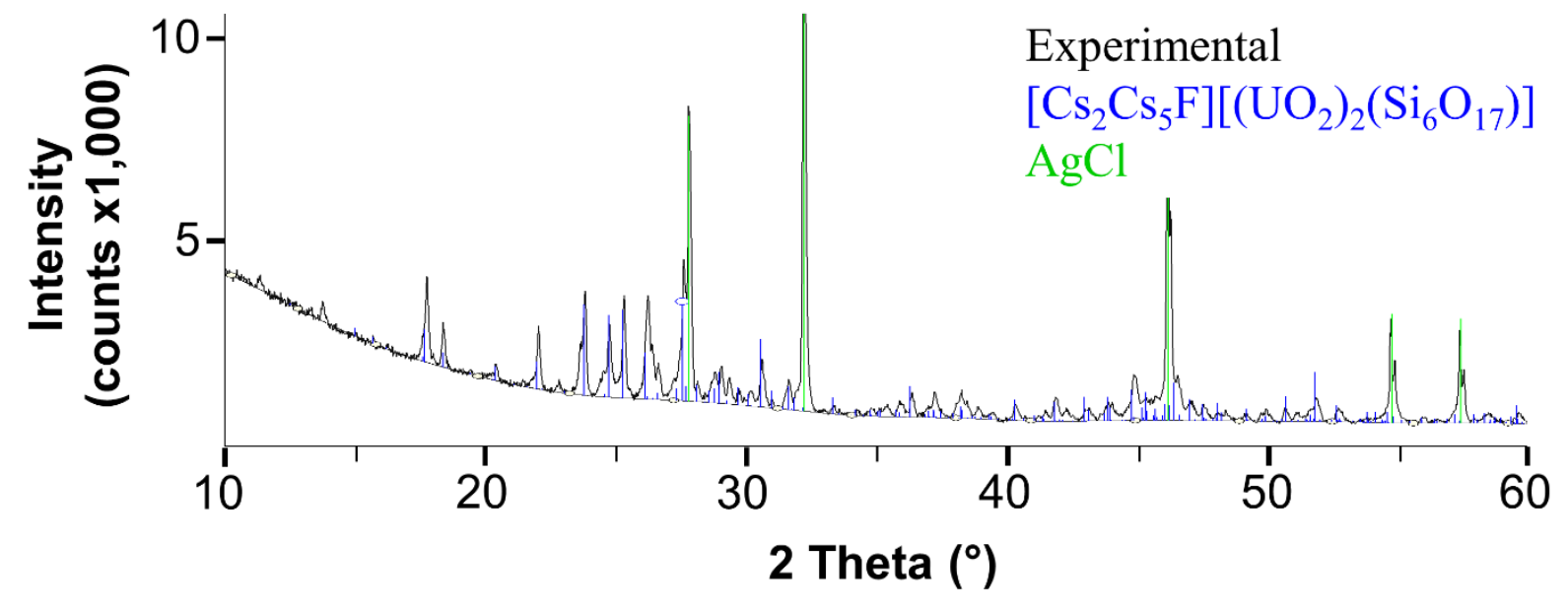


g) $\mathrm{UO}_{2}$ 1:16

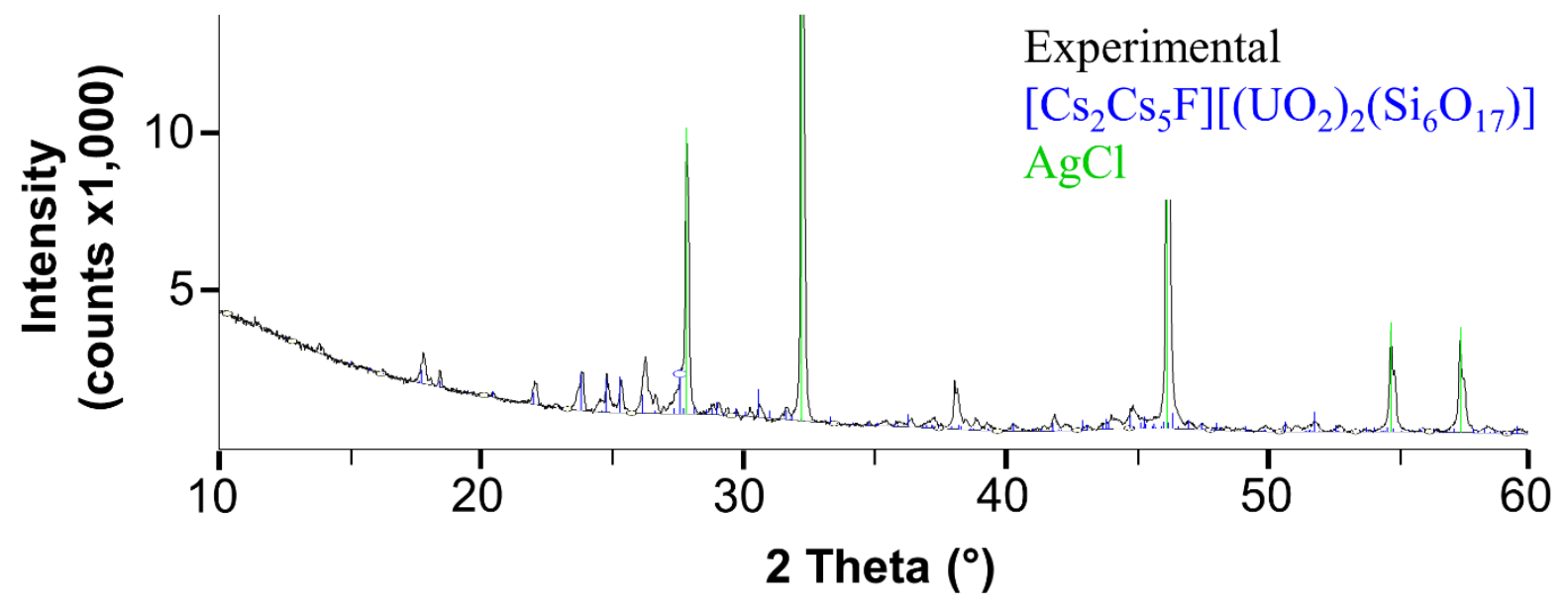

h) $\mathbf{U F}_{4} \mathbf{1 : 1}$

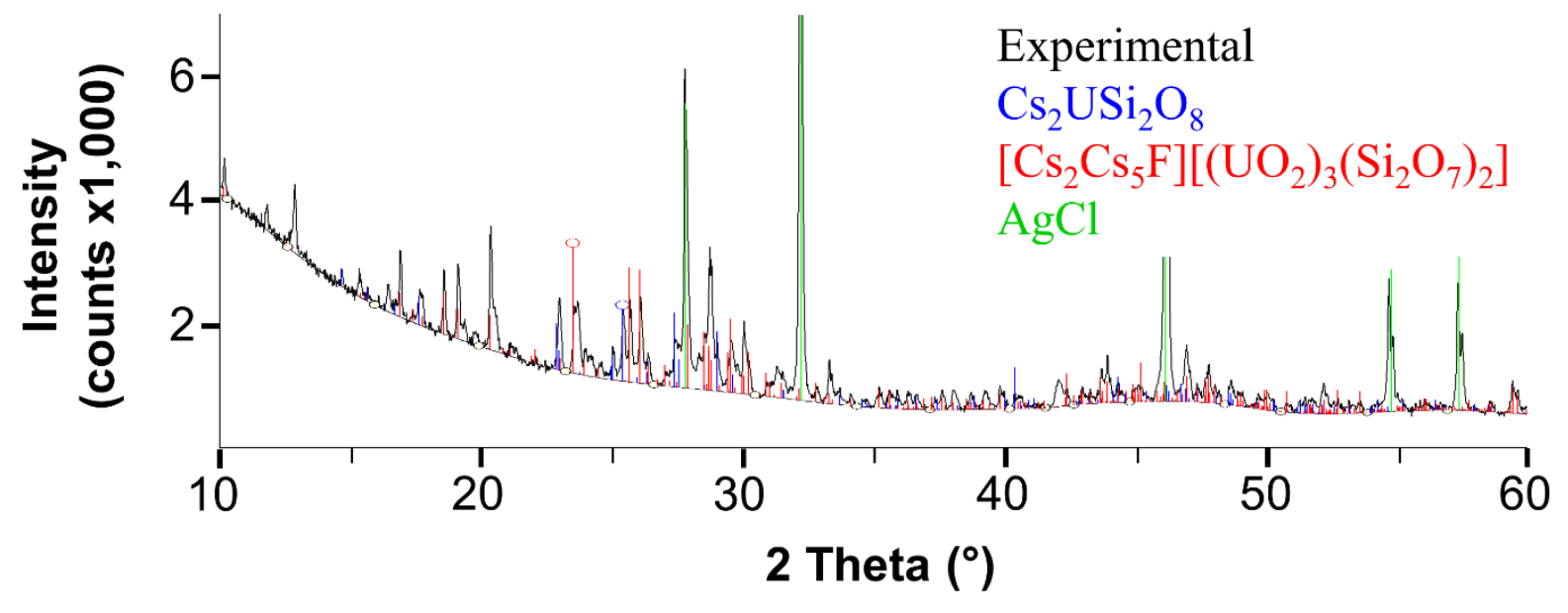

i) $\mathrm{UF}_{4}$ 1:2

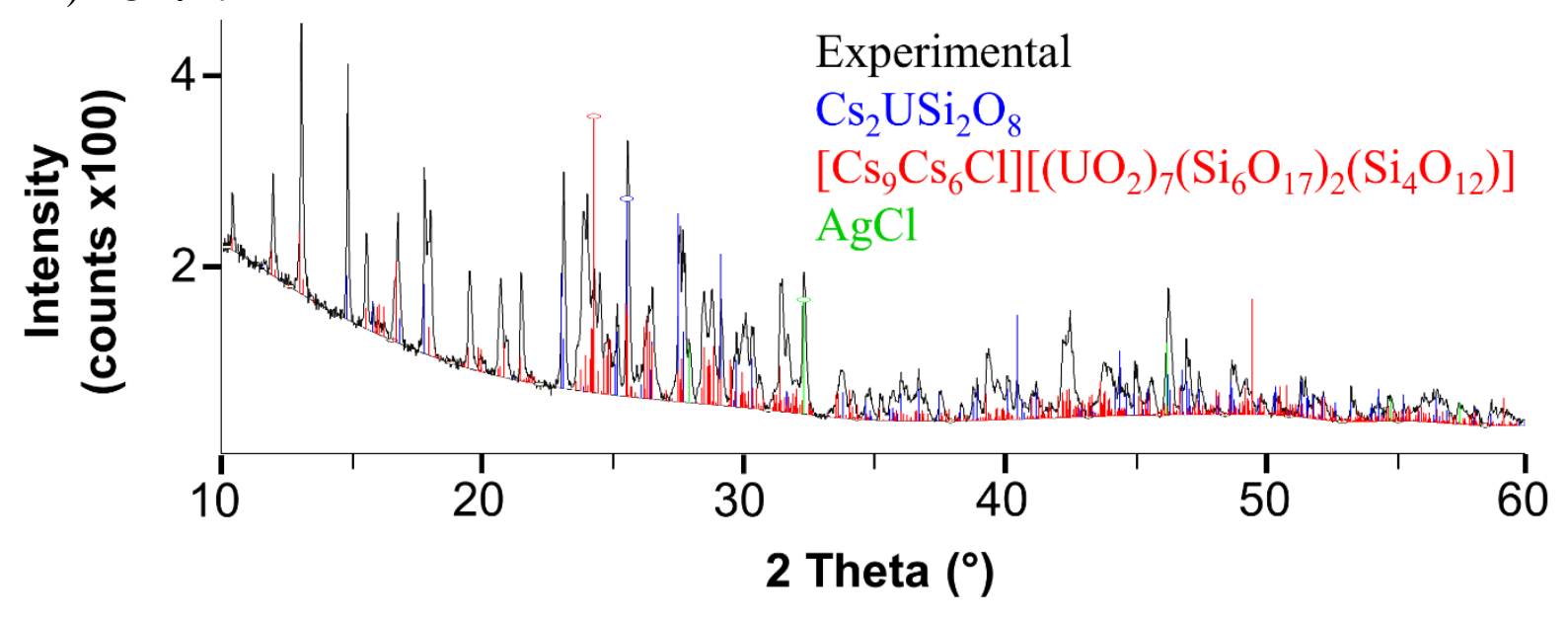


j) $\mathbf{U F}_{4} \mathbf{1 : 3}$

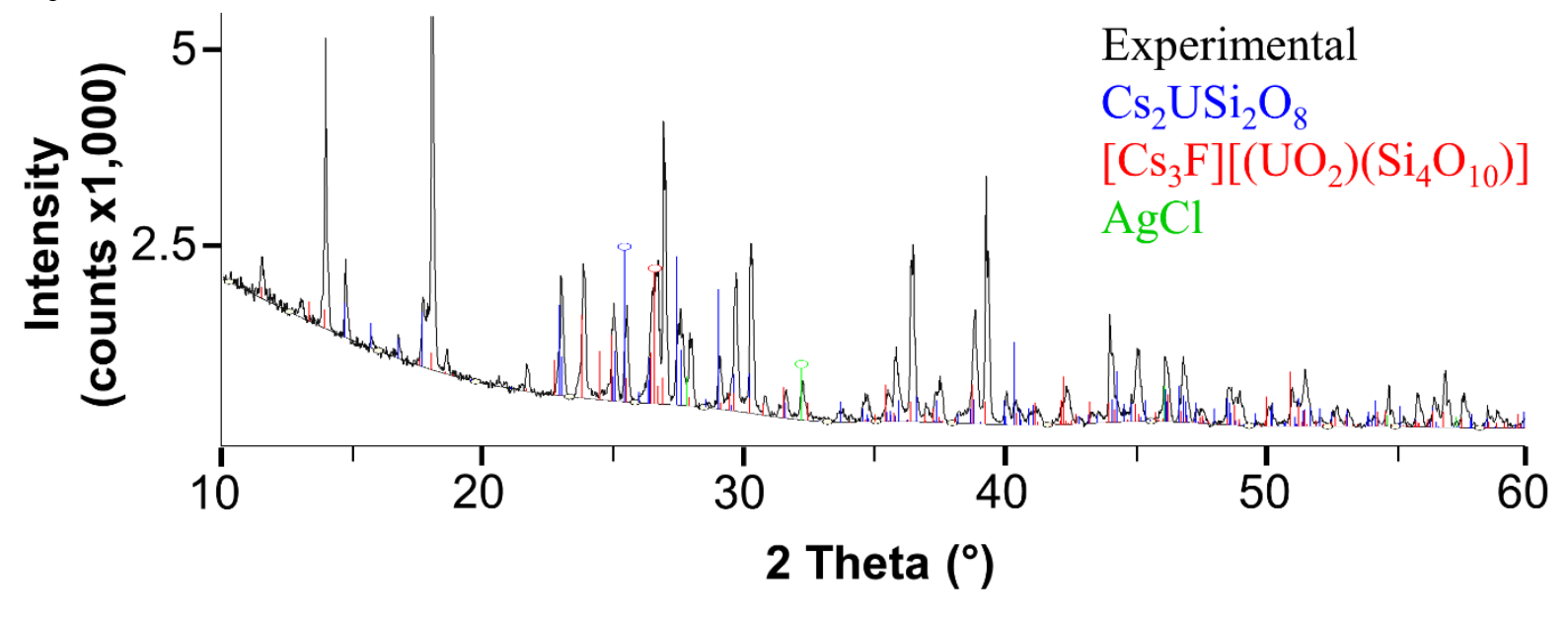

k) $\mathbf{U F}_{\mathbf{4}} \mathbf{1 : 4}$

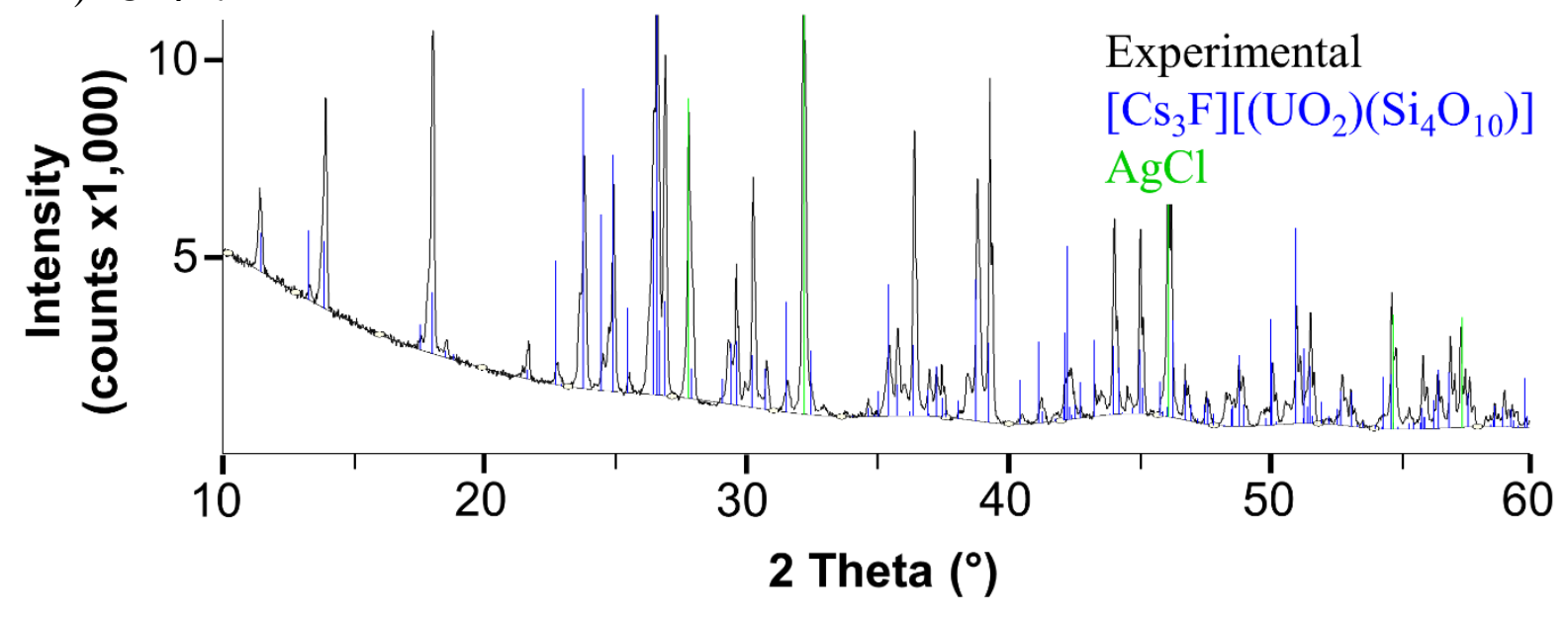

1) $\mathrm{UF}_{4} \mathbf{1 : 8}$

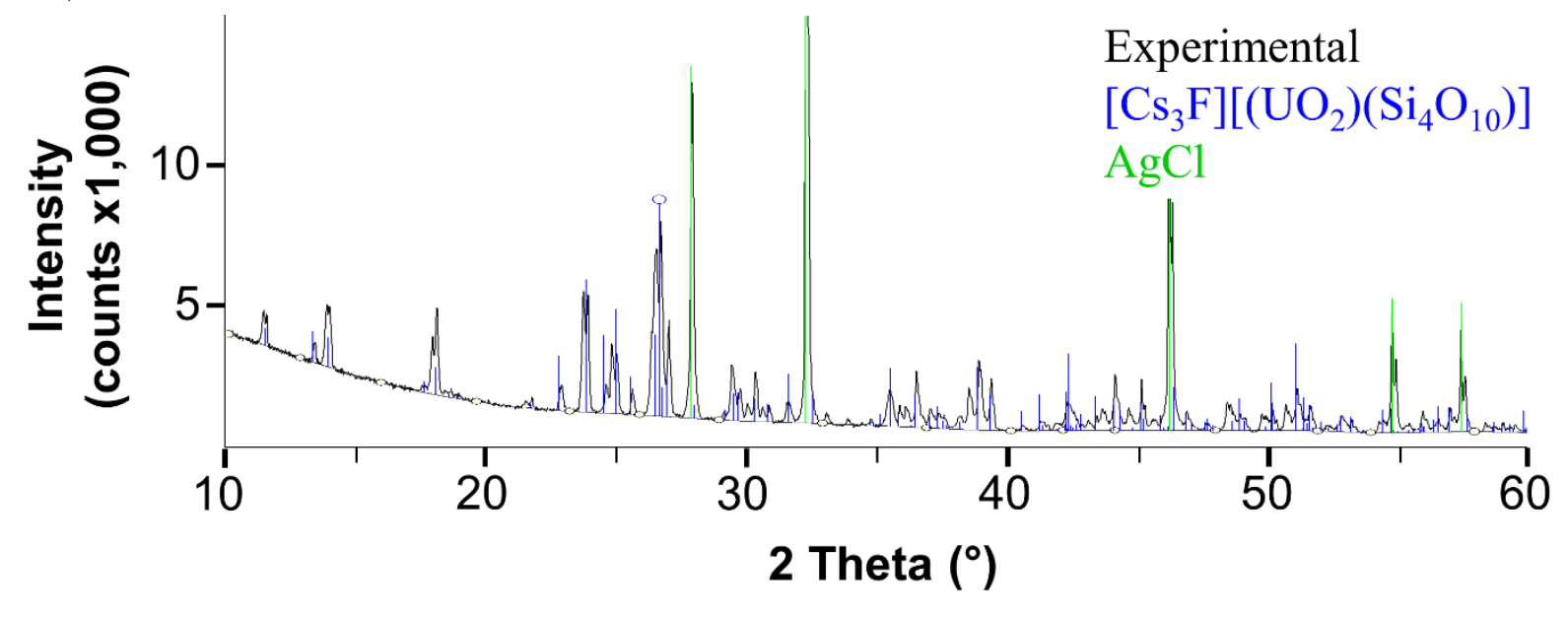


m) $\mathbf{U F}_{\mathbf{4}} \mathbf{1 : 1 2}$

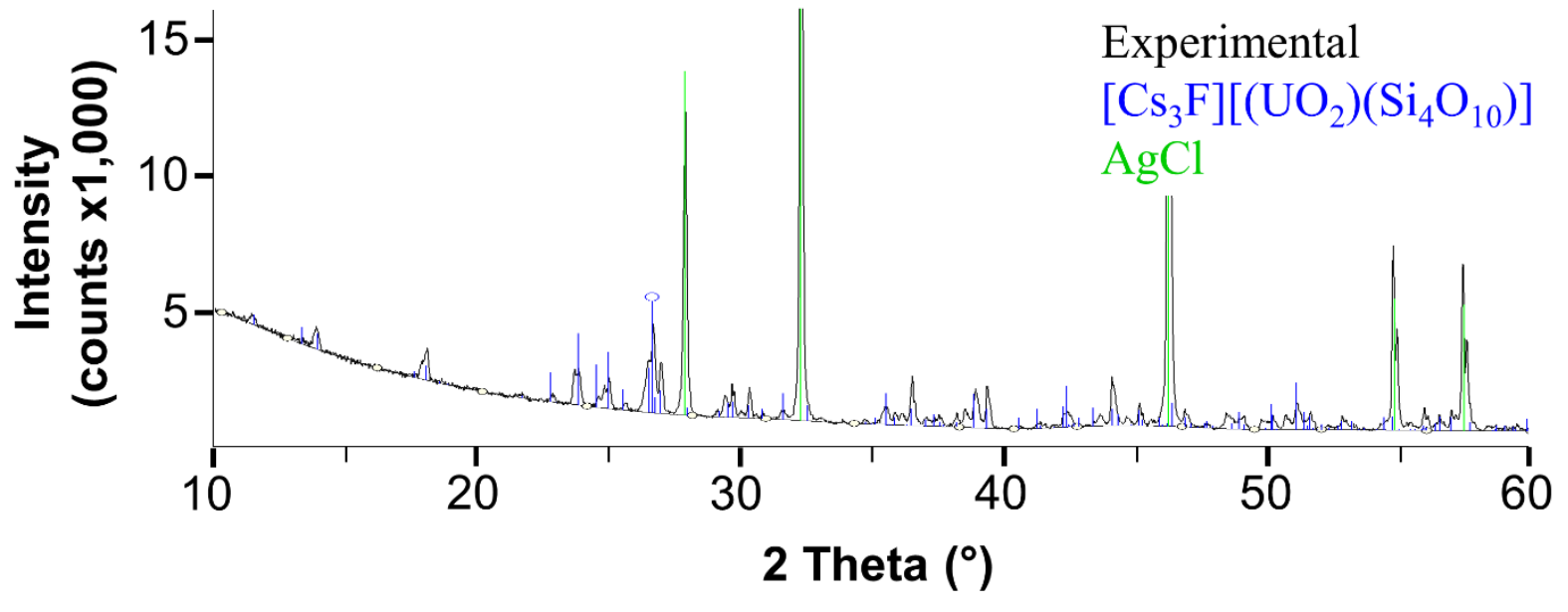

n) $\mathbf{U F}_{4} \mathbf{1 : 1 6}$

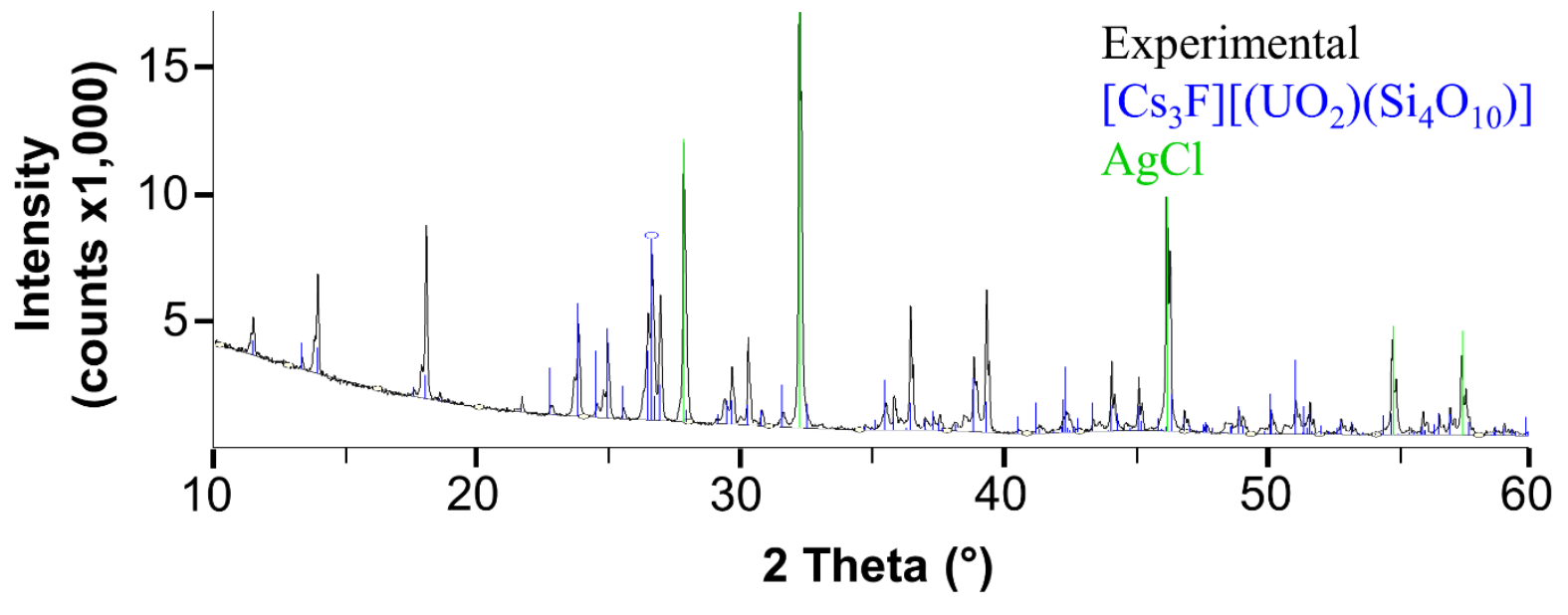

Figure S2 Powder diffraction pattern for $\left[\mathrm{Cs}_{3} \mathrm{~F}\right]\left[\left(\mathrm{UO}_{2}\right)\left(\mathrm{Si}_{4} \mathrm{O}_{10}\right)\right](\mathbf{1})$.

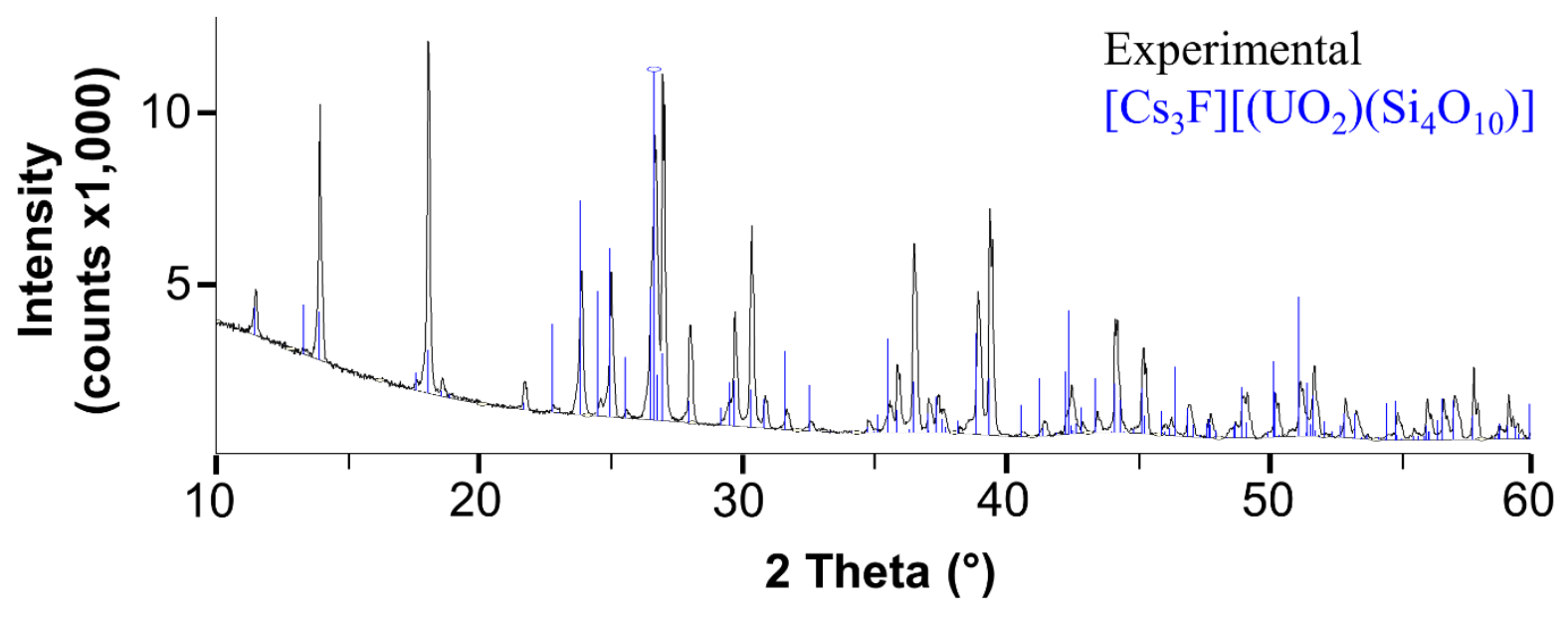


Figure S3 Further structural figures for $\left[\mathrm{Cs}_{2} \mathrm{Cs}_{5} \mathrm{~F}\right]\left[\left(\mathrm{UO}_{2}\right)_{2}\left(\mathrm{Si}_{6} \mathrm{O}_{17}\right)\right](3)$ showing the $\mathrm{Si}_{6} \mathrm{O}_{17}$-uranyl connectivity (left) and the channel formed by the 14-member ring (right).
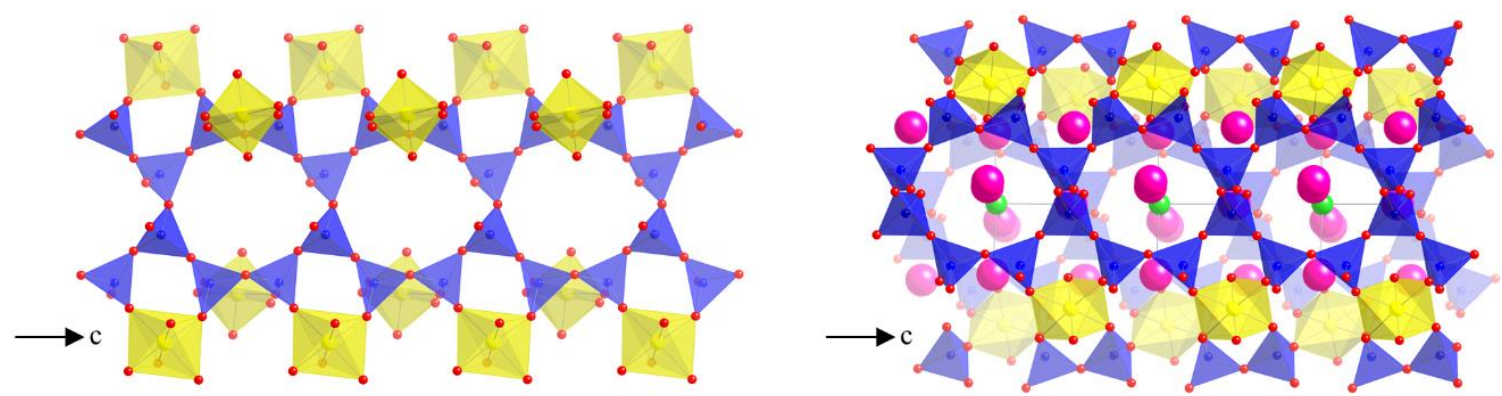


\section{References:}

(1) Shashkin, D. P.; Lur'e, E. A.; Belov, N. V. Kristallografiya 1974, 19, 958-963.

(2) Cesbron, F.; Ildefonse, P.; Sichere, M.-C. Mineral. Mag. 1993, 57, 301-308.

(3) Demartin, F.; Gramaccioli, C. M.; Pilati, T. Acta Crystallogr. C 1992, 48, 1-4.

(4) Lee, C.-S.; Wang, S.-L.; Chen, Y.-H.; Lii, K.-H. Inorg. Chem. 2009, 48, 83578361.

(5) Chen, Y.-H.; Liu, H.-K.; Chang, W.-J.; Tzou, D.-L.; Lii, K.-H. J. Solid State Chem. 2015, 236, 55-60.

(6) Liu, C.-L.; Liu, H.-K.; Chang, W.-J.; Lii, K.-H. Inorg. Chem. 2015, 54, 81658167.

(7) Morrison, G.; Tran, T. T.; Halasyamani, P. S.; zur Loye, H.-C. Inorg. Chem. 2016, 55, 3215-3217.

(8) Wang, X.; Huang, J.; Liu, L.; Jacobson, A. J. J. Mater. Chem 2002, 12, 406-410.

(9) Read, C. M.; Smith, M. D.; Withers, R.; zur Loye, H.-C. Inorg. Chem. 2015, 54, 4520-4525.

(10) Chen, C.-S.; Kao, H.-M.; Lii, K.-H. Inorg. Chem. 2005, 44, 935-940.

(11) Lee, C.-S.; Wang, S.-L.; Lii, K.-H. Chem. J. of Chin. Univ. 2011, 32, 605-608.

(12) Plaisier, J. R.; Ijdo, D. J. W.; de Mello Donega, C.; Blasse, G. Chem. Mater. 1995, 7, 738-743.

(13) Chen, C.-S.; Chiang, R.-K.; Kao, H.-M.; Lii, K.-H. Inorg. Chem. 2005, 44, 39143918.

(14) Huang, J.; Wang, X.; Jacobson, A. J. J. Mater. Chem 2003, 13, 191-196.

(15) Plášil, J.; Fejfarová, K.; Čejka, J.; Dušek, M.; Škoda, R.; Sejkora, J. Am. Mineral. 2013, 98, 718-723.

(16) Burns, P. C.; Olson, R. A.; Finch, R. J.; Hanchar, J. M.; Thibault, Y. J. Nucl. Mater. 2000, 278, 290-300.

(17) Liu, H.-K.; Chang, W.-J.; Lii, K.-H. Inorg. Chem. 2011, 50, 11773-11776.

(18) Fejfarová, K.; Plášil, J.; Yang, H.; Čejka, J.; Dušek, M.; Downs, R. T.; Barkley, M. C.; Škoda, R. Am. Mineral. 2012, 97, 750-754. 